\title{
Lucky imaging and speckle discrimination for the detection of faint companions with adaptive optics
}

Szymon Gladysz, Julian Christou, Nicholas Law, Richard Dekany, Michael Redfern, et al.

Szymon Gladysz, Julian Christou, Nicholas Law, Richard Dekany, Michael Redfern, Craig Mackay, "Lucky imaging and speckle discrimination for the detection of faint companions with adaptive optics," Proc. SPIE 7015, Adaptive Optics Systems, 70152H (10 July 2008); doi: 10.1117/12.788442

Event: SPIE Astronomical Telescopes + Instrumentation, 2008, Marseille, France 


\title{
"Lucky imaging" and speckle discrimination for the detection of faint companions with adaptive optics
}

\author{
Szymon Gladysz ${ }^{* a}$, Julian Christou ${ }^{b}$, Nicholas Law $^{c}$, Richard Dekany ${ }^{c}$, \\ Michael Redfern ${ }^{\mathrm{a}}$, Craig Mackay ${ }^{\mathrm{d}}$ \\ ${ }^{a}$ Department of Experimental Physics, National Univ. of Ireland, Galway, Ireland; \\ ${ }^{\mathrm{b}}$ Division of Astronomical Sciences, National Science Foundation, \\ 4201 Wilson Blvd., Arlington, VA 22230, USA; \\ 'Caltech Optical Observatories, California Institute of Technology, Pasadena, CA 91125, USA; \\ ${ }^{\mathrm{d}}$ Institute of Astronomy, University of Cambridge, Madingley Rd., Cambridge, CB3 0HA, UK
}

\begin{abstract}
We have analyzed the application of frame selection ("lucky imaging") to adaptive optics (AO), short-exposure observations of faint companions. We have used the instantaneous Strehl ratio as an image quality metric. The probability density function (PDF) of this quantity can be used to determine the outcome of frame selection in terms of optimizing the Strehl ratio and the peak-signal-to-noise-ratio of the shift-and-add image. In the presence of static speckles, frame selection can lead to both: improvement in resolution - as quantified by the Strehl ratio, as well as faint signal detectability - given by the peak-signal-to-noise-ratio. This theoretical prediction is confirmed with real data from AO observations using Lick Observatory's 3m Shane telescope, and the Palomar Observatory's 5m Hale telescope. In addition, we propose a novel statistics-based technique for the detection of faint companions from a sequence of AOcorrected exposures. The algorithm, which we call stochastic speckle discrimination, utilizes the "statistical signature" of the centre of the point spread function (PSF) to discriminate between faint companions and static speckles. The technique yields excellent results even for signals invisible in the shift-and-add images.
\end{abstract}

Keywords: adaptive optics, image processing, astronomy, faint companions, exoplanets

\section{INTRODUCTION}

Frame selection or "lucky imaging" is an image-sharpening technique first proposed by Fried ", as a technique for improving the resolution of astronomical images. The method is an extension of the classic shift-and-add technique (SAA), which uses a large number of short exposures instead of one long exposure. These short exposures are then spatially translated so that the image motion component of the blurring process is removed. Frame selection aims at achieving near-diffraction-limited resolution by co-adding only those frames which satisfy some image quality criterion. Nowadays "lucky imaging" is commonly used in the visible $(0.5-1 \mu \mathrm{m})$ thanks to the availability of charge coupled devices (CCDs) with negligibly low readout noise ${ }^{2}$. This has prompted our study of the closed-loop adaptive-optics (AO) short-exposure images to investigate how effective this approach would be for compensated imaging. In this paper we present the results of our theoretical modelling and observations carried out at the Lick Observatory over a period of three years. We also describe observations using the experimental LAMP instrument at the Palomar observatory. LAMP is an electron-multiplying (EMCCD)-based fast frame rate camera that was built for fast frame rate experiments behind the PALMAO adaptive optics system ${ }^{3}$. Frame selection has been traditionally used as a sharpening technique. Here we show how it can be used as a detection tool in the presence of static speckles, which limit the detectability of faint companions.

In the process of modelling the PDF of the instantaneous Strehl ratio we have noticed a significant difference between the on-axis and off-axis intensity statistics. This difference has been utilized as a way to discriminate between static speckles and faint companions ${ }^{4}$. In this paper we introduce a simpler version of the stochastic speckle discrimination

\footnotetext{
*Szymon.Gladysz@nuigalway.ie; phone+35391492824
}

\author{
Adaptive Optics Systems, edited by Norbert Hubin, Claire E. Max, Peter L. Wizinowich, \\ Proc. of SPIE Vol. 7015, 70152H, (2008) \\ 0277-786X/08/\$18 $\cdot$ doi: $10.1117 / 12.788442$
}

Proc. of SPIE Vol. $701570152 \mathrm{H}-1$ 
(SSD) approach, where only the moments of intensity and not the full intensity PDF need to be estimated. We apply the algorithm to simulated and real faint companions, and demonstrate a significant improvement in sensitivity without adding any new instrumentation.

\section{OBSERVATIONS}

\subsection{Lick Observatory}

In our system-characterization campaign at the Lick Observatory we observed over 50 single stars and 7 double stars using the natural guide star adaptive optics system on the $3 \mathrm{~m}$ Shane Telescope. Closed loop images of bright sources were obtained using the high-speed sub-array mode with a size of $64 \times 64$ pixels of the $256 \times 256$ pixel IRCAL camera ${ }^{5}$. This corresponds to field size of $4.864 \times 4.864$ arcseconds. The integration times used in the observations were either $22 \mathrm{~ms}$ or $57 \mathrm{~ms}$ and we collected $\sim 10^{4}$ exposures per target. All data were obtained in the two-micron $(K)$ band where the diffraction-limit is 151 mas so that the PSF was effectively Nyquist-sampled. The observations were made close to the zenith and using the highest possible closed-loop frame rate. The individual short exposures were registered with subpixel accuracy to produce SAA images. Measured Strehl ratios in these SAA images were in the range 0.25 - 0.55. Details of the data reduction and computation of the Strehl ratio are presented in the previous paper ${ }^{6}$.

\subsection{Palomar Observatory}

At the $5 \mathrm{~m}$ Hale telescope at Palomar observatory we used the LAMP ${ }^{3}$ Lucky Imaging camera behind the PALMAO AO system to image a variety of targets. The observations used here were of single and double stars imaged in $10 \mathrm{~nm}$ bandpasses centred around $670 \mathrm{~nm}$ and $950 \mathrm{~nm}$, with pixel sizes corresponding to Nyquist sampling. Datasets were taken at 20-50 frames per second (with negligible inter-frame deadtime) and consisted of several thousand frames. The seeing during these observations (spread over several nights) varied between 0.8 and 1.2 arcseconds; long-exposure Strehl ratios behind the $\mathrm{AO}$ system were around 0.05 for the $670 \mathrm{~nm}$ observations and around 0.25 for the $950 \mathrm{~nm}$ data.

\section{3. "LUCKY IMAGING" WITH ADAPTIVE OPTICS}

\subsection{Introduction to the analytical modelling of "lucky imaging"}

The standard implementation of the "lucky imaging" method consists of three main steps ${ }^{7}$ :

- selection of the image quality metric suitable for the type of observations,

- calculation of this metric for each frame; the dataset is then sorted in the order of increasing image quality,

- selection of the frames corresponding to some arbitrary quality threshold (expressed in terms of the defined metric); these frames are then re-centred and co-added to produce the SAA image.

We set out to predict the performance of the method in terms of the Strehl ratio and the peak-signal-to-noise-ratio of the SAA image after frame selection. The stochastic character of the technique suggests probabilistic analysis, whereby the average numbers of frames satisfying the definition of "good quality" are given by the PDF of the metric. In this paper we adopt the Strehl ratio, $S$, as the image quality metric.

To predict the Strehl ratio of the SAA image after frame selection it is enough to realize that the Strehl ratio of a sum of images is the average Strehl ratio:

$$
S_{\mathrm{SAA}}\left(S_{t}\right)=\frac{1}{\int_{S_{t}}^{1} p(s) d s} \int_{S_{t}}^{1} p(s) \cdot s d s
$$

where $S_{t}$ is the selection threshold, i.e. only those frames with their Strehl ratios higher than $S_{t}$ are co-added, and $p(s)$ is the distribution (PDF) of the Strehl ratio.

Equation (1) conveys the notion that selecting the best frames must lead to an increase in the resulting SAA Strehl ratio, which is the expected value of the Strehl ratio distribution if all frames are selected. This expected value is the second integral in equation (1). When only a fraction of the total number of frames is used to construct the SAA image, the 
initial probabilities $p(s)$ have to be re-normalized. This can be explained by the following example. If one throws a fair dice with the sides denoted by $1,2, \ldots, 6$ the expected value is 3.5 . If one wants to obtain the expected value for the case when only numbers 5 and 6 are considered, then, using the old probabilities equal to $1 / 6$, they would obtain the answer 1.8 which is clearly wrong. The reason is that the probabilities have to be re-normalized to sum - or integrate in case of continuous random variables - to one. Using new probabilities, equal to $1 / 2$, one gets the correct answer of 5.5. Therefore initial PDF values have to be divided by the area under the distribution curve starting at a given Strehl threshold $\left(S_{t}\right)$. This is the first integral in equation (1).

The peak-signal-to-noise ratio (PSNR) will be proportional to the Strehl ratio:

$$
\operatorname{PSNR}_{\mathrm{SAA}}\left(S_{t}\right) \propto \frac{S_{\mathrm{SAA}}\left(S_{t}\right)}{\left(\sigma_{\text {ind }}^{2} \cdot n \int_{S_{t}}^{1} p(s) d s+\sigma_{\text {stat }}^{2} \cdot\left(n \int_{S_{t}}^{1} p(s) d s\right)^{2}+S_{\mathrm{SAA}}\left(S_{t}\right)\right)^{1 / 2}}
$$

where $n$ is the total number of images taken. Hence $n \int_{S_{t}}^{1} p(s) d s$ is the average number of frames with Strehl ratios higher than a chosen threshold $S_{t}$. Three components of noise are expressed in the denominator: independent realizations of noise $\sigma_{\text {ind }}^{2}$ (sky, readout or random speckle noise) wherein the total variance varies linearly with the number of co-added frames; static speckles $\sigma_{\text {stat }}^{2}$ wherein the variance scales quadratically as more images are superimposed, and the Poisson noise where the variance is equal to the signal itself. Here, the shift-and-add algorithm is assumed to give a cumulated image - not the average one - as is the case in practice. The contribution of the static speckles to the overall noise variance is important in the detection of faint companions near bright sources which is the topic of this paper.

The "Strehl-to-Noise-Ratio" given in equation (2) has to be converted to the standard form of the PSNR relating peak intensity to noise variance for long exposures. We therefore develop a relationship between the Strehl ratio and the peak intensity which can be used in the analytical modelling. We assumed that the detector being used is under-sampled. The pixel scale is set to $2.44 \lambda / \mathrm{D}$. The results do not change significantly when smaller pixels are used. It is known that the first Airy ring contains $84 \%$ of the total incoming light ${ }^{8}$. This amount will be decreased in a blurred PSF and the reduction in intensity is quantified by the Strehl ratio:

$$
I=S \cdot 0.84 \cdot l
$$

The total flux in detected counts per frame $(l)$ is determined by the star's magnitude and the exposure time. The flux in the SAA image will be linearly scaled by the number of co-added (not averaged) frames. The peak intensity is then obtained using the equation:

$$
I_{\mathrm{SAA}}=S_{\mathrm{SAA}} \cdot 0.84 \cdot l \cdot n \cdot \int_{S_{t}}^{1} p(s) d s
$$

Replacing $S_{\mathrm{SAA}}$ with $I_{\mathrm{SAA}}$ and assuming some levels of independent and static noise contributions in equation (2), the proportionality becomes equality and the equation becomes the standard form of the PSNR.

The role of the Strehl ratio PDF has to be emphasized here. Knowledge of this PDF together with variances of random and static noise components is enough to completely determine the outcome of the method. Therefore it is crucial to understand the SR variability over short timescales. Two approaches have been developed to model the Strehl ratio PDF 6,9 and in the next section we give a brief introduction to the subject.

\subsection{Probability density function of the instantaneous Strehl ratio}

The development of models for the Strehl ratio PDF was motivated by the observation that histograms of AO instantaneous Strehl ratio, defined as the normalized peak intensity of a single frame, displayed negative skewness (lowend tails $)^{6}$. This stands in contrast with the uncompensated pure speckle case, where the Strehl ratio histograms have an 
excess of high values ${ }^{7}$. These high-quality outliers form a justification for "lucky imaging", so when faced with the shortage of such outliers in AO-assisted imaging, one had to ask question whether frame selection makes sense with corrected frames. As stated in the previous section, Strehl ratio PDF is key to predicting the performance of the method.

This PDF can be modelled by relating the Strehl ratio to the atmospheric coherence scale, $r_{0}$, or wavefront phase variance $\sigma^{2}$, using the Maréchal approximation:

$$
S=e^{-\sigma^{2}}
$$

where the expected phase variance can be traced back to $r_{0}$ via the error budget equations ${ }^{6}$. Given the monotonic functional relationship in equation (5) the PDF of either phase variance or $r_{0}$ can be translated to the Strehl ratio PDF. For example, assuming log-normal short-exposure $r_{0}$ :

$$
p\left(r_{0}\right)=\frac{1}{\rho \sqrt{2 \pi}\left(r_{0}-\theta\right)} \exp \left(-\frac{\left(\ln \left(r_{0}-\theta\right)-\zeta\right)^{2}}{2 \rho^{2}}\right)
$$

where $\theta$ is the location parameter of the distribution, $\zeta$ is the scale parameter, and $\rho$ is the shape parameter, one arrives at the following expression for the Strehl ratio PDF:

$$
p(s)=\frac{C}{s} s^{{ }^{8 / 3}}\left(s^{\prime}-\theta\right) \frac{2 \zeta-\ln \left(s^{\prime}-\theta\right)-2 \rho^{2}}{2 \rho^{2}}
$$

where

$$
C=3 \exp \left(-\zeta^{2} / 2 \rho^{2}\right) / 5 A \rho \sqrt{2 \pi} \quad \text { and } \quad s^{\prime}=(-\ln s / A)^{-3 / 5}
$$

and $A$ is a constant containing parameters of the $\mathrm{AO}$ system like inter-actuator spacing, and the site-specific atmospheric parameters like the Greenwood frequency. In the following analysis we employed the characteristics of the Lick AO system and the IRCAL camera (optical transmission, quantum efficiency, etc.) operating in the $K$-band ${ }^{10}$. In Figure 1 the PDF given by equation (7) is plotted for three values of $r_{0}$. The PDF for the low-correction regime is positively-skewed as observed empirically ${ }^{3}$. Skewness shifts towards negative values in the moderate and high-correction regime, which is precisely what has been noticed in infra-red observations ${ }^{6}$.

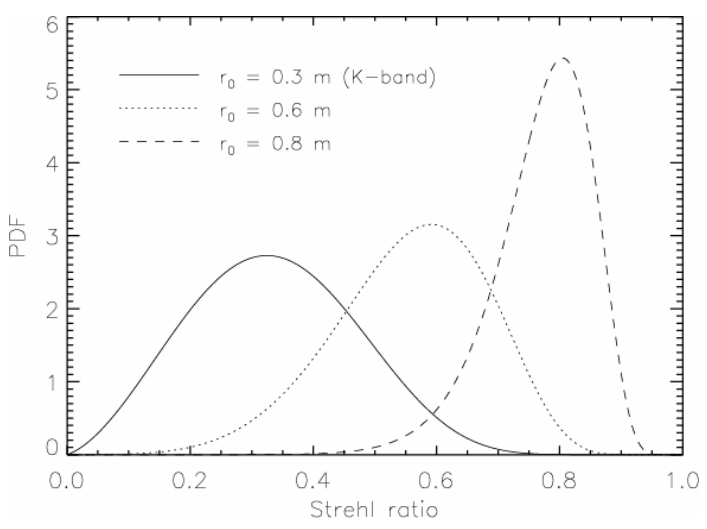

Fig. 1. Distributions of the instantaneous Strehl ratio for three levels of turbulence strength. 


\subsection{Predicted performance of the frame selection}

Given the analytical form of the Strehl ratio PDF we plot the functions $S_{\mathrm{SAA}}\left(S_{t}\right)$ and $\operatorname{PSNR}_{\mathrm{SAA}}\left(S_{t}\right)$ vs. the selection threshold $S_{t}$. This is shown in Figure 2.
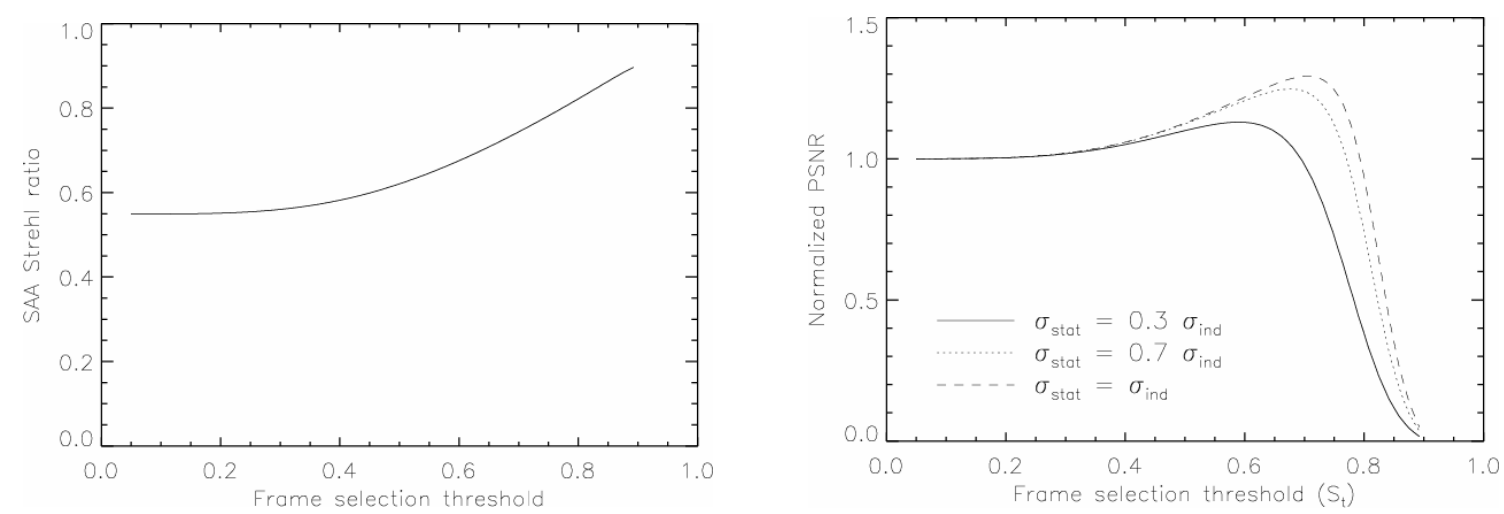

Fig. 2. Left: Strehl ratio vs. selection threshold. Right: PSNR vs. selection threshold. Median $r_{0}(2.2 \mu \mathrm{m})$ was set to $0.6 \mathrm{~m}$.

The right plot in Figure 2 is easy to interpret: the more stringent the selection rate, the better the final Strehl ratio. Surprisingly the PSNR curve possesses a maximum suggesting it is possible to improve PSNR by choosing an optimal selection rate. This is caused by the static noise contribution. In such a situation the PSNR does not improve dramatically with time ${ }^{11}$ because every new frame adds precisely the same noise to the accumulated image. This effect can be circumvented by only co-adding the best frames. The optimal number of co-added frames (the position of the maximum of the PSNR curve) depends on the relative strength of the static noise component. Figure 2 suggests that the process of optimal frame selection could lead to improvement in both: the Strehl ratio and the PSNR. We tested this prediction on images of binary stars observed at Lick and Palomar observatories.

\section{4 "Lucky imaging" with the Lick and the Palomar PSFs}

In this section we present the results of frame selection applied to images of close binaries. The presented cases cover the $0.5-1$ arcsec range of separations, and 3-4.5 range of magnitude difference. The StarFinder PSF-fitting algorithm ${ }^{12}$ was used to extract relative photometry and astrometry of the systems based on calibration PSFs obtained before or after science targets.

HD 8799, Lick Observatory

Central panel in Figure 3 shows the SAA image of the binary star HD $8799\left(m_{V}=4.83, m_{K}=3.93\right)$. The measured magnitude difference and separation were $\Delta m_{K}=3.64$ and $\theta=0.67$ ", respectively. In the left panel we artificially saturate the pixels used in the noise estimation process. PSNR was calculated with the following formula:

$$
\operatorname{PSNR}=\frac{I_{\text {peak }}-E\left(I_{\text {ring }}\right)}{\left(\operatorname{Var}\left(I_{\text {ring }}\right)+I_{\text {peak }}\right)^{1 / 2}}
$$

where $I_{\text {peak }}$ corresponds to the measured maximum intensity for the companion in the SAA image and $I_{\text {ring }}$ is the set of intensities in the ring (annulus) with the same radius as the star-companion separation; $E()$ and $\operatorname{Var}()$ denote the mean value and variance. Static and random noise contributions cannot be separated in the real images therefore they are measured simultaneously in $\operatorname{Var}\left(I_{\text {ring }}\right)$. The second term in the denominator corresponds to the Poisson noise. 

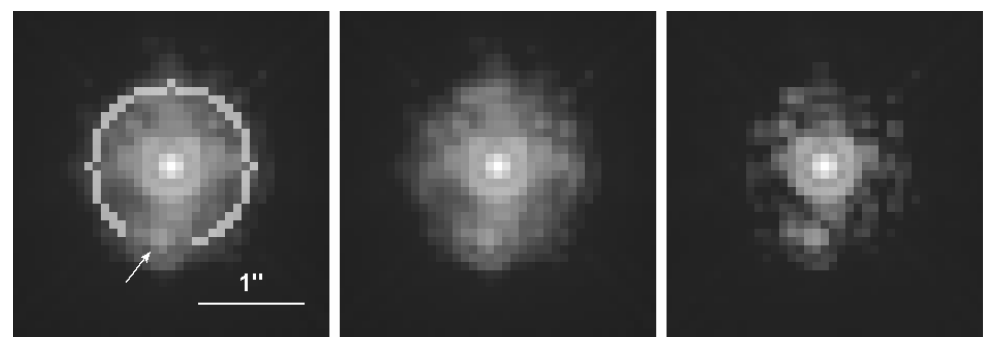

Fig. 3. Left: SAA image of the binary star HD 8799 displayed in square-root intensity scale with the artificially enhanced annulus used for noise estimation. Centre: SAA image. Right: Optimal image after frame selection.

The range of measured peak intensities for the main star was divided into fifty thresholds. The PSNR and peak intensity of the companion in the "lucky imaging" SAA images corresponding to these thresholds are plotted in Figure 4. Strehl ratio could not be used as the selection threshold because its measurement requires flux normalization between the SAA image and the diffraction-limited PSF. In close binary stars the companion is located inside this area.
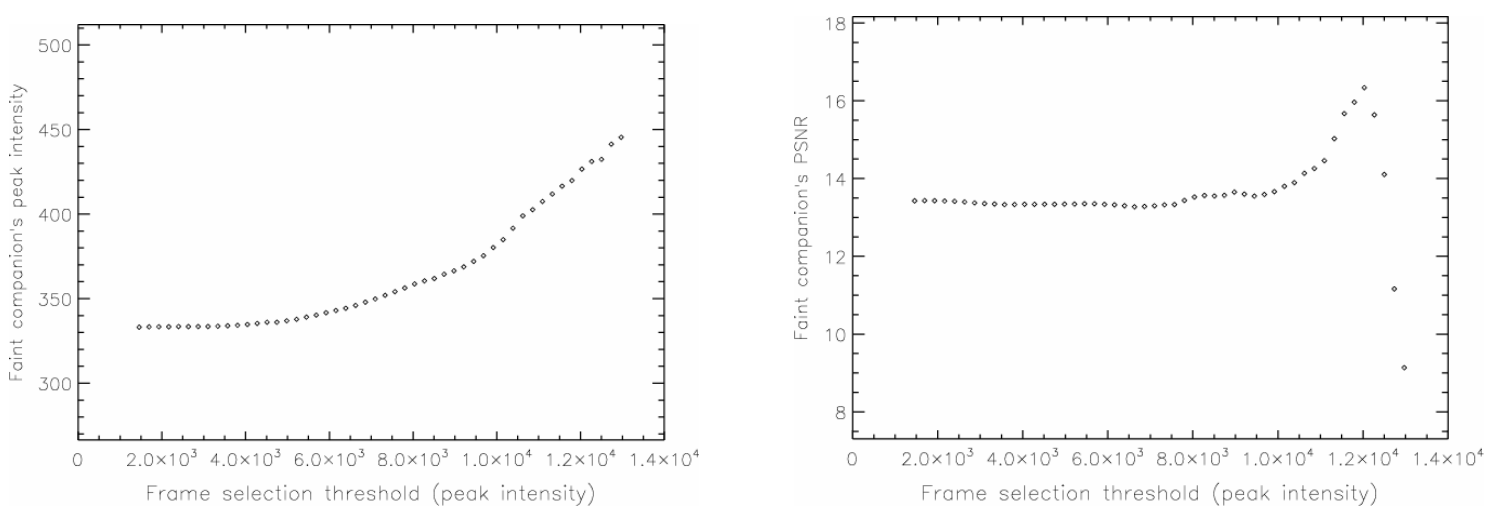

Fig. 4. Left: Peak intensity of the companion in HD 8799 measured as the function of the frame selection threshold. Right: PSNR vs. frame selection threshold.

We obtained excellent qualitative agreement between the theory and the observations. Both curves in Figure 4 look very similar to the corresponding curves in Figure 2. The maximum in the PSNR plot is present at the selection rate of $1 \%$. At this rate the increase in PSNR was $22 \%$ and the corresponding gain in peak intensity was $28 \%$. The SAA image after optimal frame selection is shown in the rightmost panel in Figure 3. It is significantly sharper than the SAA image with no frame selection. We point out that the image of the companion became sharper while the static speckles decreased in intensity. This is because intensity at the location of the companion has the same variability as the on-axis value.

In high-contrast $\mathrm{AO}$ observations, static speckles masquerading as real sources are the main problem. We believe that frame selection can be helpful here. If the results of this technique (Figure 4) were measured on a false source (static speckle) the predicted gain in peak intensity and PSNR would not be observed. This is why "lucky imaging" can be successfully used as a detection tool.

\section{HD 170648, Lick Observatory}

For HD $170648\left(m_{V}=8.04, m_{K}=7.21\right)$ we measured $\Delta m_{K}=3.07$ and $\theta=0.69 "$. The peak intensity and PSNR curves shown in the two right panels in Figure 5 display very similar behaviour to the one predicted by our theory (Figure 2). The optimal selection rate was $2.5 \%$. The increase in PSNR was $25 \%$ and the corresponding gain in peak intensity was $12 \%$. The visual improvement in the optimal-selection image is not as vivid as in the case of HD 8799 . 

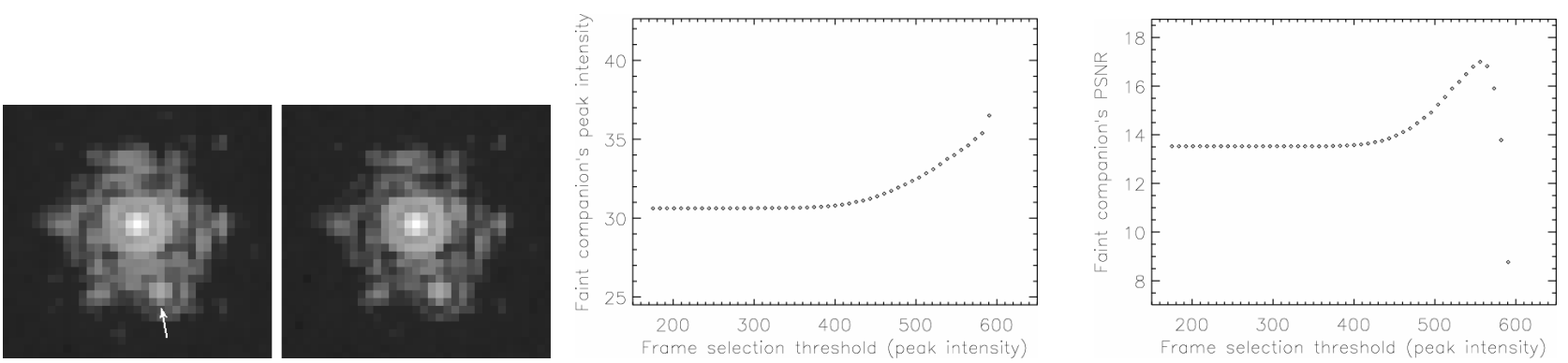

Fig. 5. Left: SAA image of the binary star HD 170648, and the optimal-selection PSF. Right: Peak intensity and the PSNR of the companion vs. selection threshold.

\section{HD 202444, Lick Observatory}

For this star $\left(\tau C y g, m_{V}=3.72, m_{K}=2.54\right)$ StarFinder yielded $\Delta m_{K}=2.26$ and $\theta=0.76 "$. Optimal selection has increased the PSNR by $31 \%$ with corresponding $39 \%$ gain in companion's peak intensity. The optimal selection rate turned out to be $1 \%$.
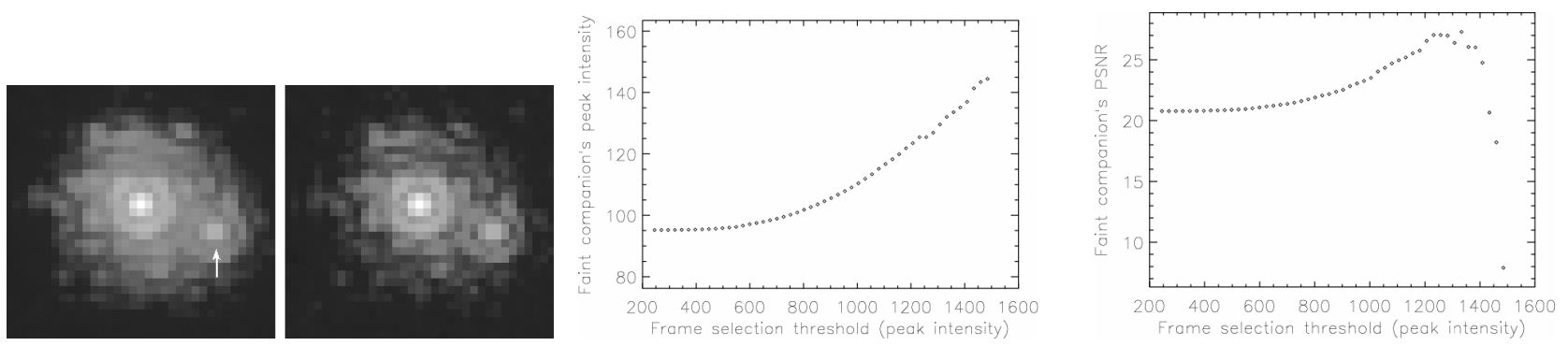

Fig. 6. Binary star HD 202444, same order as in Figure 5.

\section{$\beta C y g$, Palomar Observatory}

This double star $\left(m_{V}=3.1, m_{K}=0.39\right)$ was imaged at the wavelength of $670 \mathrm{~nm}$ using the LAMP camera at the Palomar Observatory. The rightmost panel in Figure 7 shows the SAA PSF of the system for which we calculate $\Delta m_{R}=4.3$ and $\theta$ $=0.35$ ". Even though this is a low-Strehl-ratio $(\sim 0.05)$ dataset and the static speckles contribution was less significant compared to the Lick data, we still observed an increase in the companion intensity as well as the PSNR brought upon by the optimal frame selection. PSNR was increased by $20 \%$ (40\% gain in peak intensity) at the selection rate of $10 \%$.
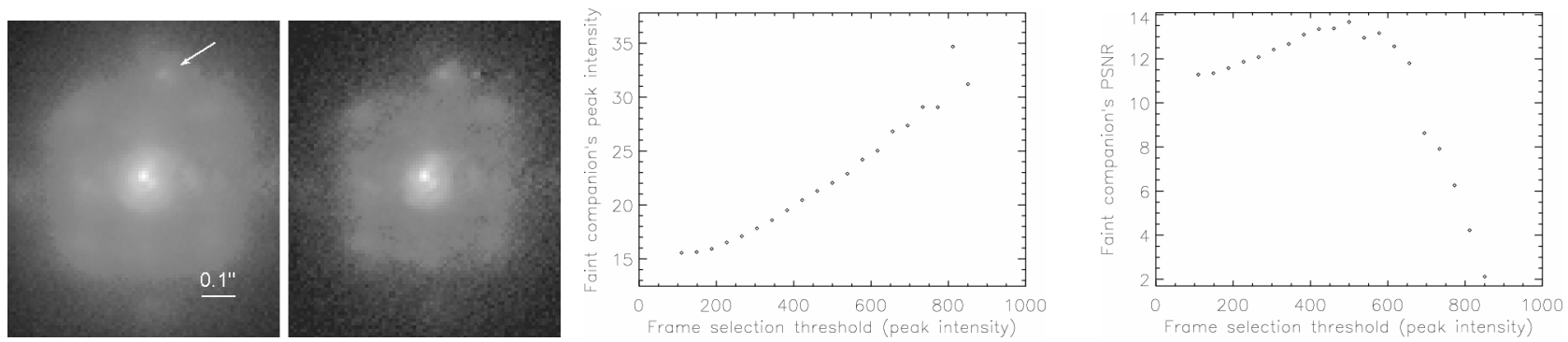

Fig. 7. Binary star $\beta$ Cyg, same order as in Figure 5.

It has to be emphasized that the frame rate of these observations was not sufficient to capture all the PSF variability at the very short timescales in the visible band. This is why we used a single-star dataset HD $192849\left(m_{V}=8.3, m_{K}=7.92\right)$ imaged with the high frame rate of $50 \mathrm{fps}$ at the wavelength of $950 \mathrm{~nm}$ to simulate a binary system with a separation of $0.14 "$ and $\Delta m_{I}=4.5$. With the correct observational setup the potential of optimal frame selection is fully realized (Figure 8). PSNR was increased by 70\% (30\% gain in peak intensity) at the selection rate of $4.5 \%$. 

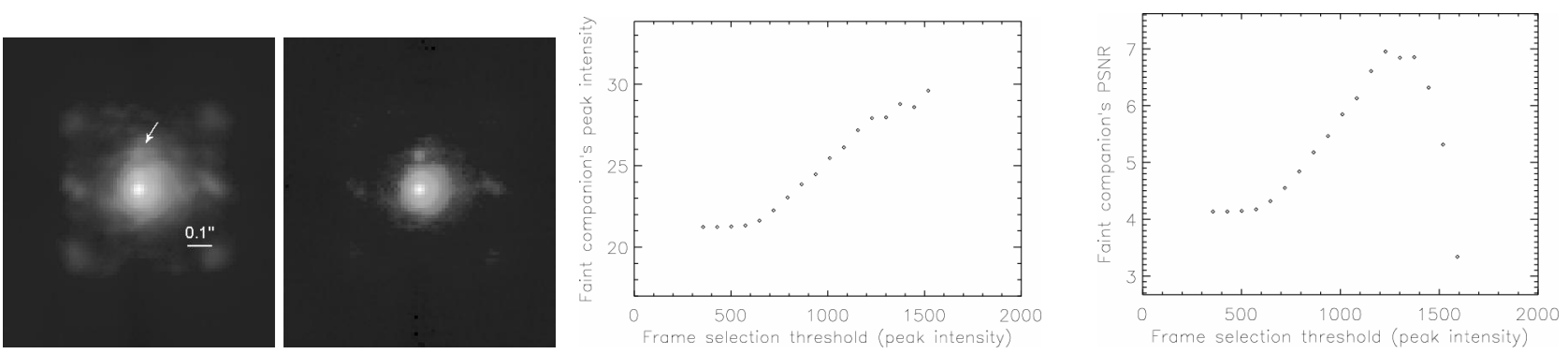

Fig. 8. Artificial binary star produced by scaling and shifting single-star dataset HD 192849, same order as in Figure 5.

\section{STOCHASTIC SPECKLE DISCRIMINATION}

\subsection{Intensity statistics in AO-corrected images}

As mentioned before, the detectability of faint companions (exoplanets and substellar companions) is limited by the presence of static speckles in the high-contrast AO observations. This particular noise contribution often arises after the wavefront sensor (non-common-path errors), so static speckles stand out against the AO-corrected halo and masquerade as faint sources. Recent papers ${ }^{13,14}$ have shown how difficult it is to make reliable detections in the presence of static speckles. This is why all planned high-contrast instruments will incorporate speckle suppression modules ${ }^{15}$

We believe that the capability of current AO systems can be greatly enhanced by exploiting the statistical information present in corrected short exposures. Just as the spectral signature of a planet is used to isolate its signal from the starlight ${ }^{15}$, so can statistical information about its intensity - which is simply the scaled and shifted copy of the on-axis intensity in an isoplanatic approximation - be utilized for the same task. The advantage of the "stochastic speckle discrimination" methods ${ }^{4}$ is that they are sensitive to any objects located close to bright stars, because these techniques only depend on the properties of the image formation process.

This section is only a brief introduction to the subject of speckle statistics. For a complete review the reader should consult earlier papers ${ }^{4,9,16}$.

The speckle intensity distribution for any location (except the centre, where the distribution is approximated by equation (7)) in the image plane is described by the modified Rician function:

$$
p(I)=\frac{1}{I_{s}} \exp \left(-\frac{I+I_{c}}{I_{s}}\right) I_{0}\left(\frac{2 \sqrt{I} \sqrt{I_{c}}}{I_{s}}\right)
$$

where $I_{c}$ corresponds to the intensity produced by the deterministic (constant) part of the wavefront, and $I_{s}$ corresponds to the halo produced by random intensity variations. $I_{0}$ is the zero-order modified Bessel function of the first kind.

The parameters $I_{c}$ and $I_{s}$ are related to the expected value $E(I)$ and variance $\sigma_{I}^{2}$ of intensity through the following equations:

$$
\begin{aligned}
& E(I)=I_{c}+I_{s} \\
& \sigma_{I}^{2}=I_{s}^{2}+2 I_{c} I_{s}
\end{aligned}
$$

PDF given by equation (10) is positively-skewed, while in the high-Strehl-ratio regime the on-axis intensity has negative skewness (Figure 1). This difference was studied by directly comparing estimates of the distributions ${ }^{4}$. This required the computation of the parameters $I_{c}$ and $I_{s}$ for the time-series at the investigated location in a peak-shifted data cube. Subsequently only time-series with similar $I_{c}$ and $I_{s}$ were compared via distribution testing to avoid false alarms. The estimation of $I_{c}$ and $I_{s}$ was done using the method of moments, whereby the unobservable population moments are equated to their sample estimators. Equation (11) is then inverted to produce estimates of $I_{c}$ and $I_{s}$ : 


$$
\begin{aligned}
& I_{s}=E(I)-\left(E(I)^{2}-\sigma_{I}^{2}\right)^{1 / 2} \\
& I_{c}=E(I)-I_{s}
\end{aligned}
$$

It was observed that at the pixel location of the artificial companions the estimation of parameter $I_{c}$ yielded values higher than for the speckles of similar magnitude. Conversely, the value of $I_{S}$ was significantly lower for the companion's peak pixel than for the speckles. This is easy to explain heuristically. On-axis intensity is the squared Fourier coefficient corresponding to the DC wavefront component, or the coherency of the wavefront. The goal of adaptive optics is to stabilize the wavefront (and Strehl ratio). $I_{c}$, which captures the amount of coherence in a wavefront, will be large at the centre of the PSF. On the other hand, the parameter $I_{s}$ is a measure of the local intensity variations. For two pixels in the focal plane with similar mean intensity, one containing the companion and the other containing speckle, the intensity variations will be larger for the latter.

With this in mind we can design image post-processing techniques which transform spatial intensity variations (SAA images) into maps of local statistics. The two transformations we found to give best results were:

$$
\begin{gathered}
I_{\mathrm{SAA}}(x, y) \mapsto \frac{I_{s}(x, y)}{I_{c}(x, y)} \\
I_{\mathrm{SAA}}(x, y) \mapsto \frac{I_{s}(x, y)}{I_{c}(x, y)} m_{3}(x, y)
\end{gathered}
$$

where $m_{3}$ is the skewness sample estimator. The latter transformation is dictated by the fact that the on-axis intensity distribution possesses negative skewness. Addition of intensities from two independent random processes, i.e. speckle and peak of the companion's PSF, leads to a convolution of the corresponding PDFs. Therefore the negative skewness of equation (7) will gradually shift towards zero, and then will become positive, when the relative magnitude of the speckle contribution increases, i.e. the observer searches for fainter and fainter companions. One should not expect a negativelyskewed intensity histogram at the location of the suspected source. Still, the skewness should be smaller than in the case of pure speckles with similar mean intensity. For real sources multiplication by skewness decreases the ratio of $I_{s}$ and $I_{c}$ even further.

The two transformations given by equations (13) and (14) lead to two new representations of an object where centres of the PSFs have low values compared to the speckles. We illustrate this effect using simulated images of a triple-star system and real images of double stars.

\subsection{Speckle discrimination tested on simulated faint companions}

In order to test the new approach we selected the highest-flux dataset from our Lick observations (star HD 216756). Even though HD 216756 is catalogued as a single star we observed a companion, or background object, 1.4" away from the star. This does not influence the presented results. Figure 9 shows the SAA image of HD 216756. Note the static speckles - some of them persisted for months.
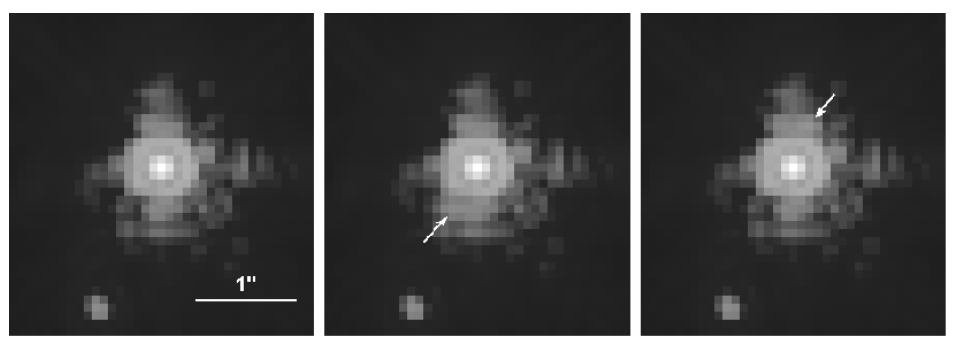

Fig. 9. Left: shift-and-add image of the star HD 216756 in square-root intensity scale. Centre: simulated companion was positioned on the zero of the speckle distribution. Right: companion located on top of a static speckle. 
A simulated faint companion $(\Delta m=4.25)$ was added to the above image five pixels down and three pixels left of the centre (separation $=0.45$ ", just below the Airy ring; central panel in Figure 9). At this location the data-cube had low intensity, so the simulated composite statistics had similar features to the central location. Figure 10 shows the direct image together with the maps corresponding to the two transformations given by equations (13) and (14). Locations dominated by Gaussian noise, where the computation of $I_{s}$ and $I_{c}$ with equation (12) proved impossible because $\sigma_{I}^{2}>E(I)^{2}$, were replaced with a constant (white pixels outside the speckle cloud in Figure 10).
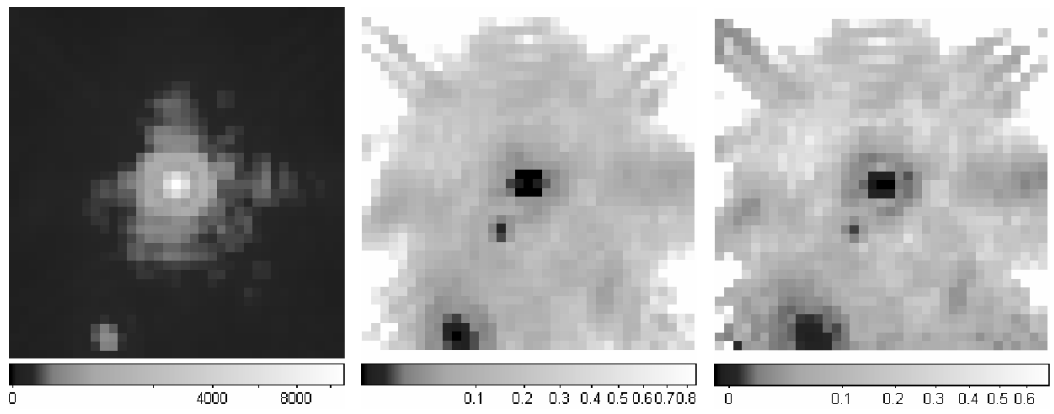

Fig. 10. Simulated triple-star system with the faint companion located on the zero of the speckle distribution: SAA image of the system (left), $I_{s} / I_{c}$ (centre), $m_{3} \cdot I_{s} / I_{c}$ (right).

While only the two bright stars can be visually identified in the SAA image, the two transformations reveal the second companion, which can now be easily seen against the halo of speckle statistics. What is remarkable is that these transformations - especially equation (13) as shown by the central panel in Figure 10 - yield very smooth maps compared to the direct images. The values of $I_{s} / I_{c}$ and $m_{3} \cdot I_{s} / I_{c}$ seem to be spatially stationary across the focal plane. This gets rid of the static speckle problem.

Next, we positioned the faint companion on top of a static speckle (five pixels up, two pixels right of the centre; rightmost panel in Figure 9). The separation was slightly smaller, and the statistics were affected more by speckle than in the previous case, therefore the visual results were worse, as seen in Figure 11. In particular the contrast in the right panel in Figure 11 is very low compared to Figure 10.
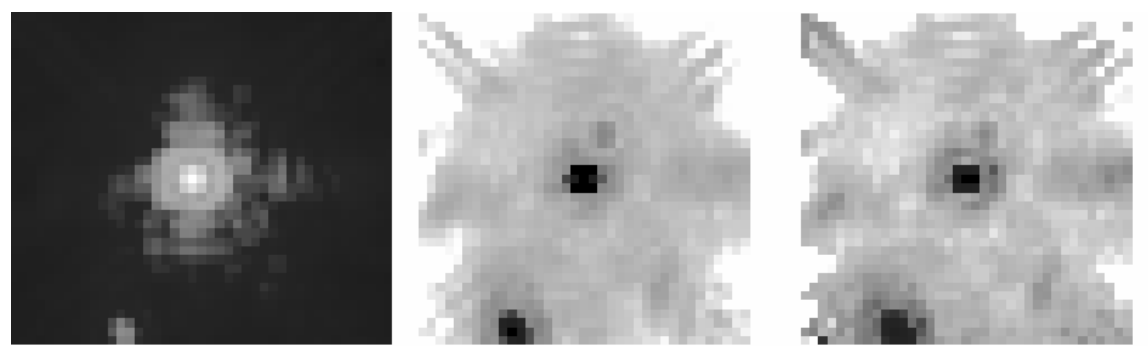

Fig. 11. Simulated triple-star system with the faint companion located on top of a static speckle just above the first Airy ring, same order as in Figure 4.

Next, we look at the observations of real double stars discussed in the previous section to see how transformations (13) and (14) work when the companion peak is not positioned on a single pixel.

\subsection{Real double stars}

Figure 12 shows the application of SSD to three double starts observed at the Lick Observatory, as well as $\beta C y g$ observed at Palomar. Again, the transformations (13) and (14) smoothed out the anisotropies present in the SAA images. For HD 8799 transformation (13) produced a very uniform map, with the exception of spiders. Visually, the faint companion is much more visible in the central and right panel as compared to the SAA image. HD 170648 had significantly lower SNR than HD 8799. This meant that many pixels had simple Gaussian statistics and therefore the statistical maps are limited spatially. There are some false-alarms in the $m_{3} \cdot I_{s} / I_{c}$ map, i.e. three pixels - two of them only partially illuminated by the companion - have very low values after the transformation. We emphasize that these false alarms are isolated as opposed to the trace of a true detection coming from all the pixels containing the companion's 
peak. For HD 202444 the two transformations yielded very similar results. $\beta C y g$ is a bright star but because the dataset had low Strehl ratio flux per pixel also turned out to be low and, just like for HD 170648, most pixels were dominated by Gaussian statistics. The companion stands out in both maps, but in the $m_{3} \cdot I_{s} / I_{c}$ map many pixels right of the centre had lower values than the PSF core, surprisingly.

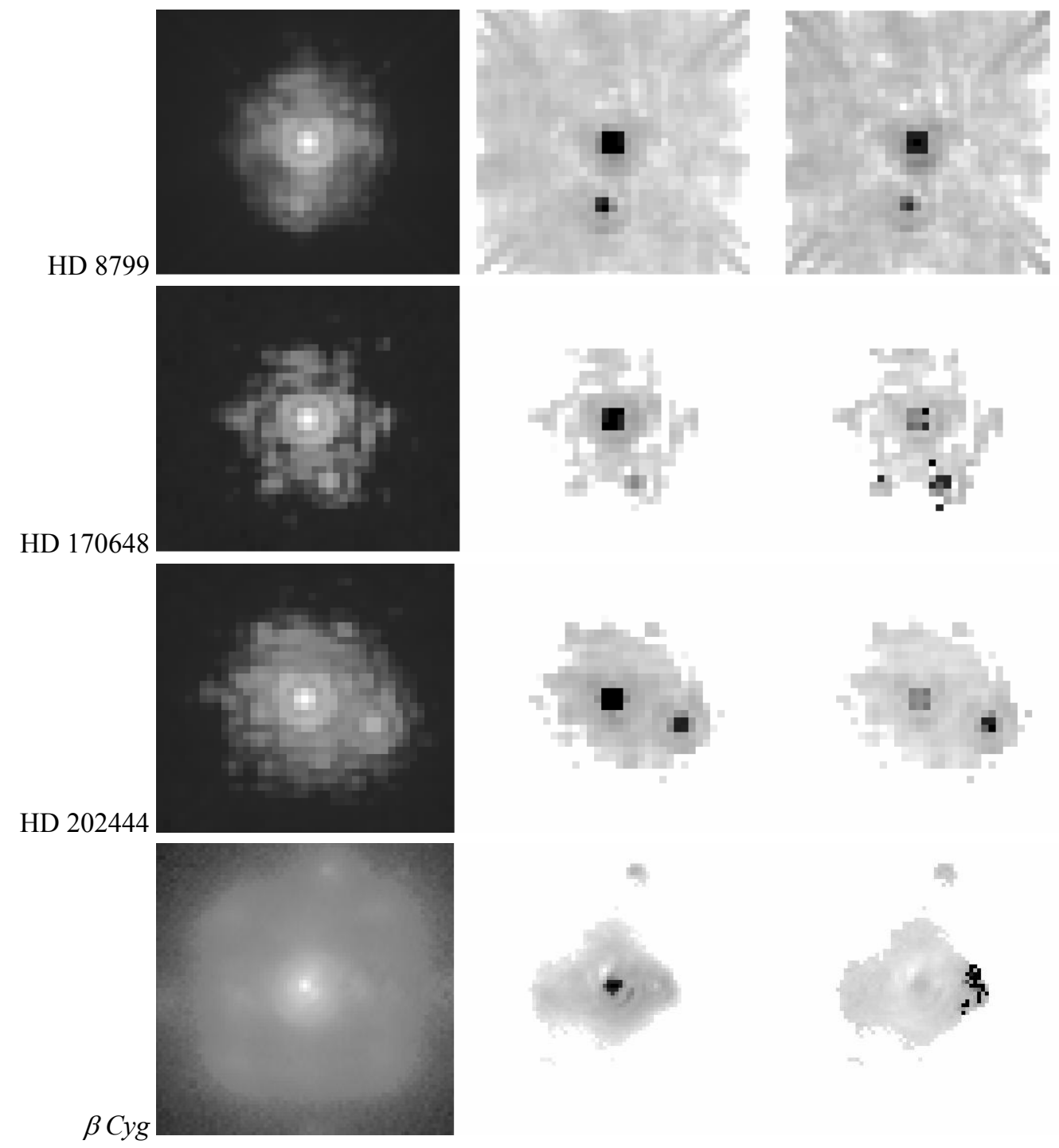

Fig. 12. Top: Binary star HD 8799: real SAA image (left), $I_{s} / I_{c}$ (centre), $m_{3} \cdot I_{s} / I_{c}$ (right). Second from top: Binary star HD 170648, same order as above. Third from top: HD 202444. Bottom: $\beta$ Cyg.

\section{CONCLUSIONS}

We have presented a novel approach to using frame selection on AO-corrected short exposures. By using optimal selection rates we were able to improve the detectability of faint companions hidden among static speckles, as predicted by theory. The method gives good results ( $20-70 \%$ increase in PSNR) across the range of Strehl ratios corresponding to low correction ( $700-900 \mathrm{~nm}$ data from the Palomar Observatory) and moderate to high correction $(2.2 \mu \mathrm{m}$ data from the Lick Observatory).

We also propose a novel statistics-based technique for the detection of faint companions. The method is shown to reveal faint companions which had been completely obscured by speckle noise in direct shift-and-add images. Stochastic speckle discrimination improved the detectability of faint sources in our Lick and Palomar observations. 
Both techniques mentioned above are reference-less, i.e. they aim at improving detectability while not requiring an estimate of the PSF to suppress speckle noise ${ }^{15}$. While such an estimate would obviously help the detection task, PSF subtraction would not remove the problem of static speckles completely, so the issues discussed here would still be relevant. The proposed methods differ in their requirements concerning the numbers of frames and their exposure times. "Lucky imaging" requires large (couple of thousands) datasets in order to apply $1-10 \%$ selection rates. The exposure times have to be as short as possible to capture the randomness of the turbulence-induced image distortions. Any averaging due to longer exposure times will lead to lower quality of the final image. While the previous approach to stochastic speckle discrimination ${ }^{4}$ also needed large numbers of images to produce an estimate of the intensity PDF, the new version presented in this paper only requires between $10^{2}$ and $10^{3}$ frames to accurately estimate the moments of intensity. The requirement of short exposure times is also released. As such the proposed algorithm can be classified as a "multiple-frame", rather than a "short-exposure" technique.

This research was supported by Science Foundation Ireland under Grants 02/PI.2/039C and 07/IN.1/I906, as well as the National Science Foundation Science and Technology Center for Adaptive Optics, which is managed by the University of California at Santa Cruz under cooperative agreement AST 98-76783. We would like to thank the staff of Lick Observatory, in particular Elinor Gates and Bryant Grigsby. In addition we would like to thank Michael Fitzgerald for information about the high-speed mode of the IRCAL camera, and Chris Dainty for support for this research.

\section{REFERENCES}

[1] Fried, D. L., "Probability of getting a lucky short-exposure image through turbulence," J. Opt. Soc. Am. 68, 16511658 (1978).

[2] Law, N. M., Mackay, C. D. and Baldwin, J. E., "Lucky imaging: high angular resolution imaging in the visible from the ground," Astronomy and Astrophysics 446, $739-745$ (2006).

[3] Law, N. M., Mackay, C. D., Dekany, R. G., Ireland, M., Lloyd, J. P., Moore, A. M., Robertson, J. G., Tuthill, P. and Woodruff, H., "Getting lucky with adaptive optics: Fast AO image selection in the visible with a large telescope," submitted to Astrophysical Journal (2008).

[4] Gladysz, S. and Christou J. C., "Detection of faint companions through stochastic speckle discrimination," Astrophysical Journal, in press (2008).

[5] Fitzgerald M. P. and Graham, J. R., "Speckle statistics in adaptively corrected images," Astrophysical Journal 637, 541-547 (2006).

[6] Gladysz, S., Christou, J. C. and Redfern, M., "Characterization of the Lick adaptive optics point spread function," Proc. SPIE 6272 (2006).

[7] Baldwin, J. E., Tubbs, R. N., Cox, G. C., Mackay, C. D., Wilson, R. W. and Andersen, M. I., "Diffraction-limited 800nm imaging with the 2.56m Nordic Optical Telescope," Astronomy and Astrophysics 368, L1-L4 (2001).

[8] Hecht, E., "Optics" (4th edition), Addison Wesley, Reading MA (2001).

[9] Gladysz, S., Christou, J. C., Bradford, L.W. and Roberts, Jr, L. C., "Temporal variability and statistics of the Strehl ratio in adaptive-optics images," submitted to Publications of the Astronomical Society of the Pacific (2008).

[10] Lloyd, J. P., Liu, M. C., Macintosh, B. A., Severson, S. A., Deich, W. T. and Graham, J. R., "IRCAL: the infrared camera for adaptive optics at Lick Observatory," Proc. SPIE 4008, 814-821 (2000).

[11] Hinkley, S., Oppenheimer, B. R., Soummer, R., Sivaramakrishnan, A., Roberts, L. C., Jr., Kuhn, J., Makidon, R. B., Perrin, M. D., Lloyd, J. P., Kratter, K. and Brenner, D., "Temporal evolution of coronagraphic dynamic range, and constraints on companions to Vega," Astrophysical Journal 654, 633-640 (2007).

[12] Diolaiti, E., Bendinelli, O., Bonaccini, D., Close, L., Currie, D. and Parmeggiani, G., "Analysis of isoplanatic high resolution stellar fields by the StarFinder code," Astronomy and Astrophysics Supplement 147, 335-346 (2000).

[13] Itoh, Y., Oasa, Y. and Fukagawa, M., "Coronagraphic Search for Extra-Solar Planets around epsilon Eri and Vega," Astrophysical Journal 652, 1729-1733 (2006).

[14] Janson, M., Brandner, W., Henning, T. and Zinnecker, H., "Early ComeOn+ adaptive optics observation of GQ Lupi and its substellar companion," Astronomy and Astrophysics 453, 609-614 (2006).

[15] Marois, C., Doyon, R., Nadeau, D., Racine, R., Riopel, M., Vallée, P. and Lafrenière, D., "TRIDENT: An Infrared Differential Imaging Camera Optimized for the Detection of Methanated Substellar Companions," Publications of the Astronomical Society of the Pacific 117, 745-756 (2005).

[16] Soummer, R., Ferrari, A., Aime, C. and Jolissaint, L., "Speckle noise and dynamic range in coronagraphic images," Astrophysical Journal 669, 642-656 (2007). 